\title{
Reducing antipsychotic drugs in stable patients with chronic schizophrenia or schizoaffective disorder: a randomized controlled pilot trial
}

\author{
Maximilian Huhn ${ }^{1,2}$ (D) Claudia Leucht ${ }^{1} \cdot$ Philipp Rothe $^{3} \cdot$ Markus Dold $^{4} \cdot$ Stephan Heres $^{5} \cdot$ Susanne Bornschein ${ }^{6,7}$. \\ Thomas Schneider-Axmann $^{8} \cdot$ Alkomiet Hasan $^{8,9} \cdot$ Stefan Leucht $^{1}$
}

Received: 23 October 2019 / Accepted: 3 February 2020 / Published online: 15 February 2020

(c) The Author(s) 2020

\begin{abstract}
As the course of schizophrenic disorders is often chronic, treatment guidelines recommend continuous maintenance treatment to prevent relapses, but antipsychotic drugs can cause many side effects. It, therefore, seems reasonable to try to reduce doses in stable phases of the illness or even try to stop medication. We conducted a 26 weeks, randomized, rater blind, feasibility study to examine individualized antipsychotic dose reduction versus continuous maintenance treatment (Register Number: NCT02307396). We included chronic, adult patients with schizophrenia or schizoaffective disorder, who were treated with any antipsychotic drug except clozapine, who had not been hospitalized in the last 3 years and who were in symptomatic remission at baseline. The primary outcome was relapse of positive symptoms. Symptom severity, social functioning and side effects were also examined as secondary outcomes. 20 patients were randomized. Relapse rates in the two groups were not significantly different. No patient had to be hospitalized. One patient in the control group dropped out. The mean reduction of antipsychotic dose in the individualized dose-reduction group was $42 \%$, however only one patient discontinued drug completely. There were no significant differences in efficacy or safety outcomes. This randomized trial provides evidence, that reduction of antipsychotic medication in chronic stable schizophrenic patients may be feasible. The results need to be confirmed in a larger trial with a longer follow-up period.
\end{abstract}

Keywords Randomized clinical trial $\cdot$ Antipsychotic maintenance treatment $\cdot$ Dose reduction $\cdot$ Chronic schizophrenia . Relapse

Electronic supplementary material The online version of this article (https://doi.org/10.1007/s00406-020-01109-y) contains supplementary material, which is available to authorized users.

Maximilian Huhn

maximilian.huhn@tum.de

1 Department of Psychiatry and Psychotherapy, Klinikum Rechts Der Isar, School of Medicine, Technical University of Munich, Ismaningerstr. 22, 81675 Munich, Germany

2 Department of Psychiatry, Psychosomatic Medicine and Psychotherapy, Social Foundation Bamberg, Teaching Hospital of the University of Erlangen, Erlangen, Germany

3 Department of Forensic Psychiatry and Psychotherapy, Günzburg District Hospital, University of Ulm, Ludwig-Heilmeyer-Straße 2, Guenzburg, Germany

4 Department of Psychiatry and Psychotherapy, Medical University of Vienna, Währinger Gürtel 18-20, Vienna, Austria
5 Klinik Nord, Isar-Amper-Klinikum München Ost, Munich, Kölner Platz, Haus 7, 80804 Munich, Germany

6 Private Practice for Psychiatry and Psychotherapy, Nymphenburger Str. 139, 80636 Munich, Germany

7 Inn-Salzach-Klinikum Wasserburg Am Inn, Gabersee 7, 83512 Wasserburg Am Inn, Germany

8 Department of Psychiatry and Psychotherapy, University Medical Hospital, Nußbaumstr. 7, 80336 Munich, Germany

9 Department of Psychiatry, Psychotherapy and Psychosomatics of the University Augsburg, Bezirkskrankenhaus Augsburg, Augsburg, Germany 


\section{Introduction}

Schizophrenia is a debilitating disorder with a frequently chronic course. Relapses are a common problem in schizophrenia which are not only a heavy burden for patients and their relatives [1], but which also cause high socio-economical costs [2]. Around $80 \%$ of patients with a first episode experience another acute episode within 5 years [3]. Therefore, prevention of relapse is a central goal of guidelinebased treatment. Antipsychotic drugs reduce relapse rates effectively from 64 to $27 \%$ after a year of treatment, as shown in a large meta-analysis [4]. Relapse rates were similar after 3-6 years [4]. Furthermore, a large cohort study has shown that long-term antipsychotic treatment prevents rehospitalization and reduces mortality in first episode patients [5]. Therefore guidelines recommend drug treatment as first option for acute and maintenance treatment of schizophrenia [6, 7]. Nevertheless, reducing antipsychotic doses in chronic schizophrenic patients is an important goal, because antipsychotics produce many side-effects. For example, metabolic side-effects can accumulate over time leading to an increased cardiovascular risk for patients with schizophrenia [8]. Furthermore, studies suggest that treatment with antipsychotics may reduce brain volume in a dose-related fashion [9]. It is therefore important to find out by how much patients can reduce antipsychotic doses or in how many patients antipsychotics can be completely withdrawn. There are three main withdrawal options. First, abrupt stopping of the antipsychotic as often done in typical relapse prevention studies, an approach, which has consistently been shown to increase relapse risk in placebo-treated patients [4]. Second, restarting antipsychotic treatment after complete withdrawal, if prodromal syndromes occur. This so-called intermittent treatment is also associated with higher relapse rates compared to continuous treatment [10]. A third option, often used in clinical routine, is the gradual dose reduction tailored to the individual patient symptoms and needs. The rationale is that there is probably a lot of interindividual variability of the doses needed for relapse prevention. Cohort studies have shown that up to one third of the patients with schizophrenia can stop medication and that these patients had more periods of recovery [11]. In a cohort study with first episode patients $30 \%$ had remission of psychotic symptoms with no current use of antipsychotic medication at 10-year follow up [12]. 50\% antipsychotic dose reduction did not increase positive symptoms and even improved cognitive function and negative symptoms in two randomized controlled trials [13, 14]. In contrast, a 14-year follow-up study in rural China found (partial) remission rates of $57 \%$ in the group taking antipsychotics compared to $30 \%$ in a group that did not [15]. Overall many patients want to reduce medication, in particular, if they suffer from severe side effects (e.g. weight gain, sedation) which reduce their quality of life. Therefore, they often reduce doses without professional support. This can lead to severe relapses and sometimes, violent acts or even death due to suicide. Thus, dose reduction seems reasonable and recommendations from the user-movement initiated a general debate on reduction of medication [16, 17], but it probably needs to be carefully adapted to the individual patient's psychopathological state. Such an approach was first systematically evaluated by Wunderink and colleagues. They tried gradual symptom guided tapering of dosage in stable first episode patients. Relapse rates were $20 \%$ higher in discontinued patients, but mostly of mild severity [18]. To examine, if gradual symptom guided dose reduction is possible in remitted chronic schizophrenic patients, we conducted a pilot feasibility study.

\section{Methods}

\section{Study design and participants}

We conducted a single-blind, randomised, controlled pragmatic feasibility trial between November 5, 2014 and 30 July 2016, in the outpatient department of the psychiatry department of the Technical University, Munich, Germany. Eligible participants were between 18 and 65 years old meeting ICD-10 criteria for schizophrenia or schizoaffective disorder. The participants had to be stable for at least 3 years, defined by no psychiatric hospitalization, and they had to be continuously treated with antipsychotic medication with no changes in the last four weeks. There was no restriction in terms of the initially used antipsychotics and their doses, except for the exclusion of clozapine. This antipsychotic is reserved for treatment-resistant patients and assumed to be associated with a high risk for rebound psychoses [19]. Moreover, patients had to be in symptomatic remission of positive symptoms as defined by the following criteria:

1. PANSS-items (positive items of the Andreasen criteria $[20])<4$ : delusions (P1), conceptual disorganisation (P2), hallucinations (P3), mannerisms and posturing (G5) and unusual thought content (G9).

2. $\mathrm{CGI}<4[21]$.

Further exclusion criteria were substance dependence other than tobacco dependency, suicidality, and initiation or dose change of antidepressants or mood stabilizers during the last six weeks before enrolment.

Our trial protocol was approved by the ethics committee of the Technical University of Munich and the trial is registered at clincialtrial.gov with the registration number: NCT02307396. All patients gave written informed consent prior to study inclusion. 


\section{Randomisation and masking}

Participants were recruited from outpatient clinics of psychiatric hospitals, ambulant psychiatrists or newsletters to patient self-help groups in the area of Munich, Germany. Eligible participants were then randomized either to the intervention (reducing of medication) or control group (no dose reduction) via a fax from the trial coordinator to A.H. who had the randomization list. The randomization list was generated by an independent psychologist using the RAND function of Microsoft Excel 2010. Only once the patient was recorded, allocation was made known to the treating psychiatrist and the participant. The ratings were done by clinicians who were blind to the allocation (C.L., P.R. and S.B.).

\section{Procedures}

In the intervention group, antipsychotic dose was gradually reduced and stopped if possible, based on the participant's psychopathological status. As a rule the initial antipsychotic dose should be reduced by $1 / 6$ every other week for the first three months, but this was adapted for each patient individually according to her/his needs and psychopathological status. So antipsychotic doses were reduced as far as possible for the first three months and then patients were followed-up with stable medication for three months. Medication in the control group was maintained. Participants were provided with two tablets of lorazepam $1 \mathrm{mg}$ as rescue medication, which were renewed, if used. Trial duration was 26 weeks with study visits every two weeks. The antipsychotic doses were adjusted by M.H., who treated the patients during the reduction. To ensure adherence to the study protocol, M.H. did a pill count at all study visits and drug levels were measured at baseline, after 12 weeks and at the endpoint. Participants were seen every two weeks and had an emergency phone number which they could call $24 \mathrm{~h}$ each day. All participating clinicians (M.H., C.L., P.R. and S.B.) had at least an experience of 6 years in clinical psychiatry.

\section{Outcomes}

\section{Primary outcome}

The primary outcome was relapse defined as a CGI $>3$ AND at least two of the following positive PANSS items $>3$ : delusions, conceptual disorganisation, hallucinations, mannerisms and posturing and unusual thought content assessed at every visit.

\section{Secondary outcomes}

The following rating scales were applied:
- "Positive and Negative Syndrome Scale" (PANSS) [22]

- "Subjective well-being under neuroleptics scale (SWN)" [23]

- "Personal and Social Performance Scale (PSP)" [24]

- "Medication Adherence Rating Scale (MARS)" [25]

- "Abnormal Involuntary Movement Scale (AIMS)" [26]

All raters were trained in the PANSS by S.H. We assessed dropouts due to any reason and due to specific reasons. Side effects were measured with the UKU-scale [27]. Olanzapine equivalence doses were calculated using the "International consensus study of antipsychotic dosing" [28].

\section{Statistical analysis}

The intention-to-treat-sample consisted of all participants randomized into the trial. The per-protocol-sample were all participants completing the trial according to protocol.

The primary dichotomous outcome was the number of patients relapsed in the intervention group compared with that in the control group. Fisher's Exact Test was applied to compare the number of relapsed patients.

As secondary endpoint time until relapse or study endpoint was graphically presented with Kaplan-Meier curves. Group differences in time to relapse were examined with the log-rank test. In addition, the median time to relapse for both study arms was determined. Cox's proportional hazard model (Cox Regression Analysis) was used to determine the effect of treatment parameters age, gender and total PANSS score at baseline (independent variable) on the outcome time to relapse (dependent variable).

Kolmogorov-Smirnov tests on normality were performed. For continuous outcomes, ANOVA was performed to compare data at baseline between the groups, if for a variable there were no significant deviations from normality assumption. Mann-Whitney $U$ tests were used, if data were tested not to be normally distributed. In case of dichotomous variables (e.g. gender), group comparisons were performed with Fisher's Exact Test or with Freeman-Halton test for $2 \times 3$ tables.

The changes in the rating scales [overall PANSS score, CGI-S, quality of life (SW-N), personal and social functional level (PSP), adherence behavior (MARS), scales for the evaluation of movement disorders (AIMS)] from time of randomization until the end of study were calculated by analysis of covariance (ANCOVA) with treatment (discontinuation or continuation of antipsychotic treatment) as a fixed factor and the respective scale value at baseline as a covariate. If the assumptions for parametric tests were violated, Mann-Whitney $U$ test or Freeman-Halton test were used as appropriate.

The occurrence of specific side effects (UKU scale) is shown descriptively and compared between the groups with 
Fisher's Exact Test. Breslow-Day tests were used to analyze, if the side effects changed significantly different between the groups from baseline until the end of the study. If there were missing values at study endpoint, last observation carried forward (LOCF) procedure was applied.

The primary statistical analysis followed the intention-totreat (ITT) principle defined as all patients being randomized. Level of significance was $\alpha=0.05$. Therefore the analyses for secondary outcomes were explorative. All tests were twotailed. SPSS version 25 was used for data analysis.

\section{Role of the funding source}

The funder had no role in study design, data collection, analysis, or interpretation or writing of the article. The corresponding author had access to all study data and had the final responsibility for the decision to submit for publication.

\section{Results}

\section{Patients}

We screened 37 patients between February 1, 2015 and December 2015 for study inclusion. Twenty-one of them were eligible and gave informed consent. One withdrew consent before randomization and was therefore not available for analysis. The initial recruitment aim was 25 patients, but the study was prematurely terminated due to lack of resources at 20 patients ( $80 \%$ of the planned sample). Eleven patients were randomised to the antipsychotic reduction group (intervention) and nine to the maintenance group (control). One subject in the intervention group refused to reduce medication and one in the control group reduced and stopped medication. Both were excluded from the per-protocol sample. The relapsed patient in the control group discontinued participation and was lost to follow-up (Fig. 1).

Eleven participants had a diagnosis of paranoid schizophrenia according to ICD-10 and nine, a diagnosis of schizoaffective disorder. The gender distribution was 6 males $/ 5$ females in the intervention group and 6 males $/ 3$ females in the control group. Intervention/control group had a mean age of 44.73/46.11 years, a mean duration of illness of 16.82/17.89 years, 3.36/4.56 previous episodes and no schizophrenic episode for 78.09/96.78 months. The antipsychotics in the intervention/control group prescribed were aripiprazole $(n=6 / n=1)$, quetiapine $(n=3 / n=5)$, olanzapine $(n=1 / n=3)$, risperidone $(n=5 / n=0)$, and perazine $(n=0 / n=1)$. In the intervention group/control group, four patients/one patient took more than one antipsychotic and the mean dose in olanzapine equivalents was $14.4 \mathrm{mg} / 9.2 \mathrm{mg}$. There was no significant difference between the characteristics of the two groups, except for family history of psychiatric illness (Table 1).

\section{Primary outcome, Cox-regression and hospitalisation}

Two patients in the control group and one in the intervention group fulfilled the relapse criteria (Fisher's exact test: $p=0.566$ ). The patient in the intervention group returned to starting dose after relapse, stabilized and did not fulfil the relapse criteria anymore. One relapse in the maintenance group was associated with social stress (illness of the mother of the patient). The patient stabilized over time without any change in medication. The other relapsed patient in the control group stopped medication against protocol, fulfilled relapse criteria afterwards, discontinued the study and was lost to follow-up. The median time to relapse was 88.5 days in the control group and 124 days in the intervention group (Log-rank: chi $^{2}=0.751, \mathrm{df}=1, p=0.386$ ) (Supplementary Fig. 1).

Using Cox-Regression to explore the influence of age, gender or PANSS-Baseline-Score on time to relapse did not yield any significant results. There was a trend for the moderator age $(p=0.059)$ (Supplementary Fig. 2), however this result was mainly caused by the youngest participant, who had the earliest relapse. This was the participant, who violated the protocol by reducing medication in the control group.

No participant had to be hospitalised during the study. Whether the one participant who dropped out was hospitalised remained unclear because he was lost to follow-up.

\section{Secondary outcomes}

There was no significant difference between groups in terms of the mean change from baseline to endpoint of PANSS total score and the PANSS subscores. Nor was there a significant difference between groups in terms of CGI-improvement, quality of life (SWN-Scale), social functioning (PSP), dyskinesia (AIMS), and adherence (MARS) (Table 2).

\section{Safety}

There were no serious adverse events or deaths. Side effects according to the UKU-scale are presented in Supplementary Table $1 \mathrm{a}+\mathrm{b}$. There was no significant difference between groups for any side effect at baseline or endpoint. In the course of the study, the number of patients with orgastic dysfunction decreased in the intervention group but increased in the control group (Breslow-Day test: $p=0.013$ ). Dream activity increased in the intervention group but decreased in the control group (Breslow-Day test: $p=0.011$ ) (details in Supplementary Table $1 \mathrm{a}+\mathrm{b}$ ). 
Fig. 1 Consort-flowchart

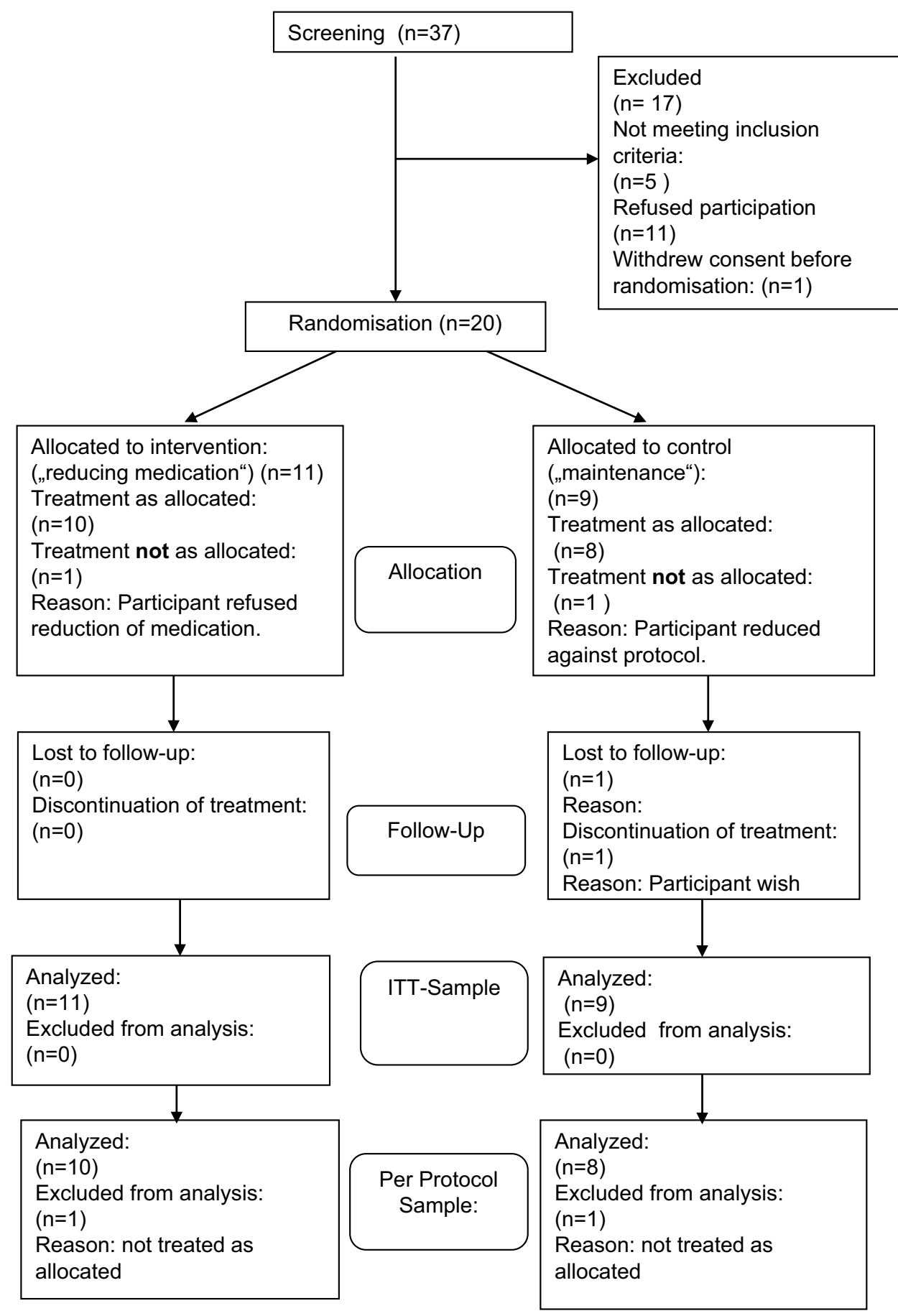

\section{Reduction of doses}

The mean reduction of antipsychotic dose in the intervention group, calculated as the arithmetic mean per patient of the reduction for each antipsychotic medicament used, was $42 \%$ (range $0-100 \%$ ) (Table 3), corresponding to a mean olanzapine equivalence dose of $9.5 \mathrm{mg} /$ day. In one patient medication was stopped completely. Reduction schemes are presented in Fig. 2. Doses were not changed in the control group except for the patient who stopped medication himself.

\section{Rescue medication}

The participant with the relapse in the intervention group took rescue medication (Lorazepam $1 \mathrm{mg}$ ) three times. Three patients in the control group took rescue 
Table 1 Baseline characteristics of the intention to treat sample

\begin{tabular}{|c|c|c|c|c|c|c|c|}
\hline \multirow[t]{2}{*}{ Characteristic } & \multicolumn{2}{|c|}{$\begin{array}{l}\text { Intervention } \\
(n=11)\end{array}$} & \multicolumn{2}{|c|}{ Control $(n=9)$} & \multirow[t]{2}{*}{$F$} & \multicolumn{2}{|c|}{ Statistic } \\
\hline & $m$ & $\mathrm{sd}$ & $m$ & sd & & df & $p$ \\
\hline Age (years) & 44.73 & 10.33 & 46.11 & 12.09 & 0.08 & 1,18 & 0.785 \\
\hline Weight $(\mathrm{kg})$ & 88.70 & 10.95 & 76.88 & 15.29 & 3.66 & 1,16 & 0.074 \\
\hline Height $(\mathrm{cm})$ & 175.89 & 5.53 & 174.13 & 7.68 & 0.30 & 1,15 & 0.592 \\
\hline PANSS overall (baseline) & 50.09 & 10.41 & 47.67 & 8.09 & 0.33 & 1,18 & 0.575 \\
\hline PANSS positive-score (baseline) & 9.27 & 2.37 & 10.11 & 2.85 & 0.52 & 1,18 & 0.481 \\
\hline PANSS negative-score (baseline) & 13.73 & 4.47 & 12.00 & 4.56 & 0.73 & 1,18 & $0.405^{\mathrm{a})}$ \\
\hline PANSS Allgemein-score (baseline) & 27.09 & 5.74 & 25.56 & 5.57 & 0.36 & 1,18 & 0.554 \\
\hline CGI (Baseline) & 2.82 & 0.41 & 3.00 & 0.00 & 1.80 & 1,18 & $0.196^{\mathrm{a})}$ \\
\hline Duration of illness (years) & 16.82 & 8.44 & 17.89 & 8.80 & 0.08 & 1,18 & 0.785 \\
\hline Number of previous hospitalisations & 3.00 & 1.67 & 3.67 & 3.28 & 0.35 & 1,18 & 0.563 \\
\hline Number of previous episodes & 3.36 & 1.75 & 4.56 & 2.83 & 1.34 & 1,18 & 0.263 \\
\hline Duration since last episode (months) & 78.09 & 45.82 & 96.78 & 57.28 & 0.66 & 1,18 & 0.428 \\
\hline Gender (male/female) & $6 / 5$ & & $6 / 3$ & & & & 0.670 \\
\hline Living alone (no/yes) & $0 / 11$ & & $1 / 8$ & & & & 0.450 \\
\hline Married or cohabiting & $7 / 4$ & & $3 / 6$ & & & & 0.370 \\
\hline Educational level (low/middle/high) & $3 / 3 / 5$ & & $2 / 0 / 7$ & & & & $0.255^{\mathrm{b}}$ \\
\hline Continuous occupation (no/yes) & $3 / 8$ & & $6 / 3$ & & & & 0.175 \\
\hline Continuous occupation ( $\leq 15 \mathrm{~h} />15 \mathrm{~h} /$ full-time $)$ & $1 / 3 / 4$ & & $1 / 0 / 2$ & & & & $0.509^{\mathrm{b})}$ \\
\hline $\begin{array}{l}\text { Diagnosis (schizophrenia/schizoaffective accord- } \\
\text { ing to ICD-10) }\end{array}$ & $7 / 4$ & & $4 / 5$ & & & & 0.653 \\
\hline Smoking (no/yes) & $5 / 6$ & & $6 / 3$ & & & & 0.406 \\
\hline Family history of psychiatric disorders (no/yes) & $5 / 6$ & & $0 / 9$ & & & & 0.038 \\
\hline
\end{tabular}

Continuous variables were analyzed using ANOVA. Categorical variables were analyzed with Fisher's exact test. $\mathrm{a}=$ Mann-Whitney $U$ test, data were not normally distributed. $\mathrm{b}=$ Freeman-Halton extension of Fisher's exact test for $2 \times 3$ field tables. $\mathrm{m}=$ mean, $\mathrm{sd}=$ standard deviation, $\mathrm{F}=$ test statistic, $\mathrm{df}=$ degrees of freedom, $p p$ value medication (Lorazepam $1 \mathrm{mg}$ ), all due to sleeping problems and inner tension. The patient who stopped medication against protocol took rescue medication (Lorazepam $1 \mathrm{mg}$ ) due to craving for alcohol.

The per-protocol analysis did not materially change the results.

\section{Power analysis for future studies}

From the present study, a power analysis for future studies can be derived, intending to show non-inferiority of medication reduction comparing to a control group maintaining at the current dosage, when from the present study relapse rates of $15 \%$ in both the intervention and the control group are estimated. Assuming $\alpha=0.05$ and a power of $1-\beta=0.8$, in both groups 26 patients will be needed to conclude that the upper limit of a one-sided $95 \%$ confidence interval will exclude a superiority in favor of the control group of more than $25 \%$ [29].

\section{Discussion}

To our knowledge, this is the first time guided antipsychotic discontinuation was examined in stable chronic schizophrenic patients in a randomized controlled design. The goal of this pilot study was to check feasibility and safety. During the trial, no patient had to be hospitalized and the single patient with a relapse in the intervention was stable at the end of the trial after increasing his dose to the original level. One participant in the control group, who violated the study protocol by abruptly stopping medication, relapsed, dropped out and was lost to follow up. The relapse of the other patient in the control group was associated with intercurrent illness of his mother, showing that even long-term stable medicated patients are vulnerable to stress and psychosocial support is crucial for a successful reduction of antipsychotic medication [30]. The mean percentage dose reduction in the intervention group was more than $40 \%$ in three months, which is quite a substantial reduction. As intended the sample consisted of patients with chronic schizophrenia or schizoaffective disorder with several previous episodes and a mean duration of illness of 15 years (Table 1). The 
Table 2 Differences between baseline and endpoint in all secondary outcomes

\begin{tabular}{|c|c|c|c|c|c|c|c|c|}
\hline \multirow[t]{2}{*}{ Differences V15 - V2 } & \multicolumn{3}{|c|}{ Intervention } & \multicolumn{3}{|c|}{ Control } & \multicolumn{2}{|l|}{ Factor group } \\
\hline & $n$ & $\mathrm{~m} /$ median & $\mathrm{sd} /-$ & $n$ & $\mathrm{~m} /$ median & $\mathrm{sd} /-$ & $\mathrm{F} / \mathrm{U} \quad \mathrm{df}$ & $p$ \\
\hline PANSS total $^{\mathrm{a}}$ (LOCF) & 11 & -3.45 & 10.61 & 9 & 3.00 & 9.59 & $1.551,17$ & 0.230 \\
\hline $\begin{array}{l}\text { PANSS positive score } \mathrm{a}^{\mathrm{a}} \\
\quad(\text { LOCF })\end{array}$ & 11 & 1.0 & - & 9 & 0.0 & - & $45.5 \quad 1$ & 0.766 \\
\hline $\begin{array}{l}\text { PANSS negative score }{ }^{\mathrm{a}} \\
\text { (LOCF) }\end{array}$ & 11 & -3.82 & 3.52 & 9 & -0.56 & 5.29 & $1.961,17$ & 0.179 \\
\hline $\begin{array}{l}\text { PANSS general score }{ }^{\mathrm{a}} \\
\text { (LOCF) }\end{array}$ & 11 & -0.55 & 6.68 & 9 & 1.33 & 2.83 & $0.161,17$ & 0.691 \\
\hline $\mathrm{SWN}^{\mathrm{b}}(\mathrm{LOCF})$ & 8 & 1.63 & 16.34 & 8 & 3.88 & 11.08 & $0.041,13$ & 0.838 \\
\hline MARS sumb & 11 & 0.27 & 1.35 & 8 & 0.38 & 0.92 & $0.221,16$ & 0.643 \\
\hline$P S P^{\mathrm{b}}$ & 11 & -10.0 & - & 8 & 10.0 & - & $24.0 \quad 1$ & 0.109 \\
\hline$A I M S^{\mathrm{a}}$ & 11 & 0.0 & - & 8 & 0.0 & - & $41.5 \quad 1$ & 0.840 \\
\hline$C G I(L O C F)$ & \multicolumn{3}{|c|}{$\begin{array}{l}\text { Improved: } n=3 \text {, unchanged: } n=7 \text {, deterio- } \\
\quad \text { rated: } n=1\end{array}$} & \multicolumn{3}{|c|}{$\begin{array}{l}\text { Improved: } n=2 \text {, unchanged: } n=6 \text {, deterio- } \\
\quad \text { rated: } n=1\end{array}$} & $-\quad-$ & $1.00 *$ \\
\hline
\end{tabular}

ANCOVA for all normally distributed outcomes (bold values), Mann-Whitney $U$ test for all others (italic values), except for *Freeman-Halton extension of Fisher's exact test

$d f$ degrees of freedom, PANSS positive and negative syndrome scale of schizophrenia, CGI clinical global impression score (V15 vs. V2: improved/unchanged/deteriorated). SWN subjective wellbeing under neuroleptics, MARS medication adherence rating scale, $P S P$ personal and social performance scale, AIMS abnormal involuntary movement scale, $\mathrm{m} /$ median mean for normally distributed and median for non normally distributed values, $n$ number of participants, $s d$ standard deviation, $L O C F$ last observation carried forward, V2 visit 2, V15 visit 15

${ }^{a}$ Negative change scores represent improvement

${ }^{\mathrm{b}}$ Positive change scores represent improvement

Table 3 Reduction of medication (dosage at study end vs. baseline) in patients of the intervention group-ITTSample

\begin{tabular}{llllll}
\hline Pat & Start medication & $\begin{array}{l}\text { Start dosage } \\
(\mathrm{mg})\end{array}$ & $\begin{array}{l}\text { End dosage } \\
(\mathrm{mg})\end{array}$ & Reduction (\%) & $\begin{array}{l}\text { Mean reduction } \\
\text { per patient }(\%)\end{array}$ \\
\hline 001 & Aripiprazole & 25 & 15 & 40 & 53.3 \\
& Quetiapine & 150 & 50 & 66.7 & \\
$002^{\mathrm{a}}$ & Risperidone depot & 25 & 25 & & 0 \\
& Quetiapine & 50 & 50 & 0.0 & \\
004 & Risperidone & & & \\
& Olanzapine & 7 & 7 & $0^{\mathrm{c}}$ & \\
005 & Aripiprazole & 1.25 & 0 & 12.5 \\
& Quetiapine & 1.25 & 15 & 25.0 & 100.0 \\
007 & Risperidone & 20 & 50 & 0.0 & 50.0 \\
011 & Aripiprazole & 50 & 0 & 100.0 & 33.3 \\
014 & Aripiprazole & 1 & 15 & 50.0 & 66.7 \\
015 & Risperidone & 30 & 20 & 33.3 & 50.0 \\
016 & Aripiprazole & 30 & 1 & 66.7 & 25.0 \\
018 & Risperidone & 3 & 7.5 & 50.0 & 75.0 \\
019 & Aripiprazole & 15 & 4.5 & 25.0 & $\mathbf{4 2 . 3}$ \\
& Mean medication reduction in & & 2.5 & 75.0 & \\
& the intervention group & & & &
\end{tabular}

${ }^{\text {a }}$ Patient refused medication reduction

${ }^{\mathrm{b}}$ Before study start patient had $1 \mathrm{mg}$ oral risperidone daily and $50 \mathrm{mg}$ risperidone depot every 2 weeks. Depot medication was switched to $6 \mathrm{mg}$ oral risperidone

${ }^{\mathrm{c}}$ After a relapse the medication had to be raised to the starting dose

${ }^{\mathrm{d}}$ Patient had $400 \mathrm{mg}$ aripiprazole every 4 weeks, which were switched to $20 \mathrm{mg}$ oral aripiprazole daily 
Fig. 2 Dose reduction from baseline to endpoint in original doses for the individual subjects

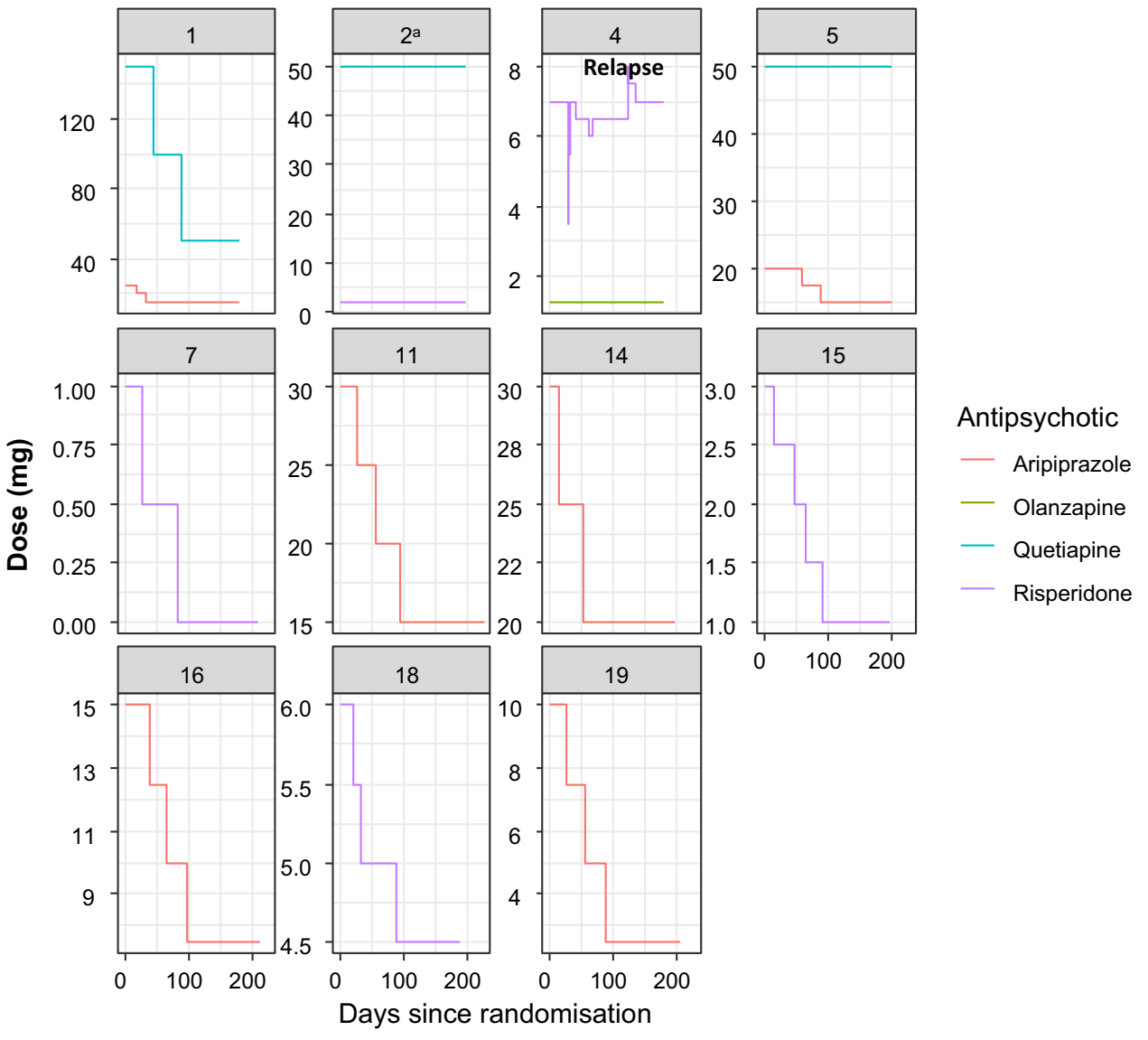

intention-to-treat population had a higher relapse rate in the control group than in the intervention group, but this was due to the abrupt withdrawal of a patient in the control group. In the per-protocol population one patient per group relapsed. None of the outcome measures was significantly different between the two groups. Although the sample size was small for definitive conclusions, these results make us confident that examining gradual and careful dose-reduction can be safely assessed in a future randomised trial. The only exceptions were increased dream activity and less orgastic dysfunction in the intervention group at endpoint.

There are limitations of our study. The sample was small, because this was a pilot study with the aim to check the feasibility of a larger trial. So all analyses should be seen as exploratory. The inclusion of patients with schizoaffective disorder, could have influenced the results, as there are data for a better prognosis for these patients [31]. Of the three participants who relapsed two had diagnosis of schizoaffective disorder and one a diagnosis of schizophrenia. Some participants had additional psychotropic drugs like antidepressants or mood stabilizers, which could have influenced psychopathology (Supplementary Table 2). But these medication had to be stable at least six weeks before enrollment and was not changed throughout the study. So possible changes in psychopathology cannot be attributed to these drugs. Even if clozapine is often used in chronic schizophrenic patients, we did not include this drug in our study. Our rational was evidence that reduction of clozapine can cause severe rebound psychoses [19]. Two included patients violated the protocol. These two patients did not reduce or maintain the dose according to the group they were allocated. Regardless, excluding them from the analysis did not change the results significantly. In time series analysis, the time until the first relapse was analyzed. Whether patients remitted again after they had relapsed was not an outcome of the study. However, the individual course of the patients is described in the section "Primary outcome, cox-regression and hospitalization" and indeed all patients remitted again. The goal to reduce $1 / 6$ of the antipsychotic dose every two weeks was ambitious, but in clinical routine patients often urge doctors to reduce the dose as fast as possible. After all we assume, that a slower dose reduction could have prevented or at least delayed the one relapse in the reduction group. The follow-up period of three months after dose reduction was short and it is possible that more relapses occur in the reduction group later on. Furthermore, quality of life and social functioning, need time to change, eventually up to 7 years as seen in the study by Wunderink [32], so possible changes may be missed due to the short follow-up period. There is also contrary evidence from a ten 
year naturalistic follow-up of a discontinuation study [33]. In the discontinuation group, more patients had a poor clinical outcome (39\%) than in the maintenance group $(21 \%)$. Also the suicide rate was higher in the discontinuation group (4\%) than in the maintenance group (2\%).

This pilot trial showed that gradual reduction of antipsychotic medication in chronic stable schizophrenic patients is feasible and can be carefully attempted under close supervision, at least in the framework of a randomized trial with study visits every two weeks. Even patients that relapsed under dose reduction stabilized after raising medication until the end of the trial and no relapsed patient had to be hospitalized. As this was a small pilot study, results need to be confirmed in a larger trial.

Acknowledgements Open Access funding provided by Projekt DEAL. We thank the Münchner Studienzentrum for support and monitoring during the trial and for administration of the trial database and Prof. Dr. Eva Meisenzahl, Dr.Ingrid Bauer, Dr. Peter-Christian Vogel, Ambulanz IAK-München-Ost, Ambulanz IAK-München-Nord, ApK München e.V. and Münchner Psychiatrie-Erfahrene (MüPE) e.V. for their help in recruiting participants.

Author Contributions SL, MD, SH and MH designed the study. SL supervised the study. MD obtained the funding. MH was the study coordinator and did the clinical treatment. CL, PR and SB did the assessments. AH did the randomization. TSA performed all statistical analyses. MH and SL analyzed and interpreted the data. MH and SL wrote the draft and the final version of the manuscript. All authors critically reviewed the report for important intellectual content and approved the final submitted version.

Funding This trial was founded by the German Research Association (Ministry of Education and Research (DO 1723/1-1)).

\section{Compliance with ethical standards}

Conflict of interest In the last 3 years $\mathrm{MH}$ has received speaker's honoraria from Janssen. SL has received honoraria as a consultant/advisor and/or for lectures from LB Pharma, Otsuka, Lundbeck, Boehringer Ingelheim, LTS Lohmann, Janssen, Johnson\&Johnson, TEVA, MSD, Sandoz, SanofiAventis, Angelini, Recordati, Sunovion, Geodon Richter. AH has received honoraria for consulting from Lundbeck, Janssen and Otsuka and for lecture from Janssen, Lundbeck and Otsuka. In the last 3 years, PR has received speaker's honoraria from Janssen. SH has received speaker honoraria from Janssen-Cilag, Eli Lilly, Sanofi-Aventis, Otsuka, Lundbeck and Johnson \& Johnson. SH has accepted travel or hospitality payment from Janssen-Cilag, SanofiAventis, Johnson \& Johnson, Pfizer, Bristol-Myers-Squibb, AstraZeneca, Lundbeck, Novartis and Eli Lilly. SH has participated in clinical trials sponsored or supported by Eli Lilly, Janssen Cilag, Johnson \& Johnson, Bristol-Myers-Squibb, AstraZeneca, Lundbeck, Novartis, Servier, Pierre Fabre, Pfizer, Organon, Roche and Merck. SH has received honoraria for participation in advisory-boards or activities as a consultant from Lundbeck, Otsuka, Eli Lilly, Roche, Teva, Janssen and Johnson \& Johnson. CL is SL spouse. For the remaining authors none were declared.

Open Access This article is licensed under a Creative Commons Attribution 4.0 International License, which permits use, sharing, adaptation, distribution and reproduction in any medium or format, as long as you give appropriate credit to the original author(s) and the source, provide a link to the Creative Commons licence, and indicate if changes were made. The images or other third party material in this article are included in the article's Creative Commons licence, unless indicated otherwise in a credit line to the material. If material is not included in the article's Creative Commons licence and your intended use is not permitted by statutory regulation or exceeds the permitted use, you will need to obtain permission directly from the copyright holder. To view a copy of this licence, visit http://creativecommons.org/licenses/by/4.0/.

\section{References}

1. Vos T, Flaxman AD, Naghavi M et al (2012) Years lived with disability (YLDs) for 1160 sequelae of 289 diseases and injuries 1990-2010: a systematic analysis for the Global Burden of Disease Study 2010. Lancet 380(9859):2163-2196. https://doi. org/10.1016/S0140-6736(12)61729-2

2. Cloutier M, Aigbogun MS, Guerin A et al (2016) The economic burden of schizophrenia in the United States in 2013. J Clin Psychiatry 77(6):764-771. https://doi.org/10.4088/JCP.15m10 278

3. Robinson D, Woerner MG, Alvir JM et al (1999) Predictors of relapse following response from a first episode of schizophrenia or schizoaffective disorder. Arch Gen Psychiatry 56(3):241-247

4. Leucht S, Tardy M, Komossa K et al (2012) Antipsychotic drugs versus placebo for relapse prevention in schizophrenia: a systematic review and meta-analysis. Lancet 379(9831):20632071. https://doi.org/10.1016/S0140-6736(12)60239-6

5. Tiihonen J, Tanskanen A, Taipale H (2018) 20-Year nationwide follow-up study on discontinuation of antipsychotic treatment in first-episode schizophrenia. Am J Psychiatry 175(8):765-773. https://doi.org/10.1176/appi.ajp.2018.17091001

6. Gaebel W, Weinmann S, Sartorius N et al (2005) Schizophrenia practice guidelines. International survey and comparison. Br J Psychiatry 187:248-255

7. Hasan A, Falkai P, Wobrock T et al (2013) World Federation of Societies of Biological Psychiatry (WFSBP) guidelines for biological treatment of schizophrenia, part 2: update 2012 on the long-term treatment of schizophrenia and management of antipsychotic-induced side effects. World J Biol Psychiatry 14(1):2-44. https://doi.org/10.3109/15622975.2012.739708

8. de Hert M, Detraux J, van Winkel R et al (2011) Metabolic and cardiovascular adverse effects associated with antipsychotic drugs. Nat Rev Endocrinol 8(2):114-126. https://doi. org/10.1038/nrendo.2011.156

9. Ho B-C, Andreasen NC, Ziebell S et al (2011) Long-term antipsychotic treatment and brain volumes: a longitudinal study of first-episode schizophrenia. Arch Gen Psychiatry 68(2):128137. https://doi.org/10.1001/archgenpsychiatry.2010.199

10. Sampson S, Mansour M, Maayan N et al (2013) Intermittent drug techniques for schizophrenia. Cochrane Database Syst Rev (7):CD006196. 10.1002/14651858.CD006196.pub2

11. Harrow M, Jobe TH, Faull RN (2012) Do all schizophrenia patients need antipsychotic treatment continuously throughout their lifetime? A 20-year longitudinal study. Psychol Med 42(10):2145-2155. https://doi.org/10.1017/S00332917120002 20

12. Wils RS, Gotfredsen DR, Hjorthøj C et al (2017) Antipsychotic medication and remission of psychotic symptoms 10 years after a first-episode psychosis. Schizophr Res 182:42-48. https://doi. org/10.1016/j.schres.2016.10.030 
13. Takeuchi H, Suzuki T, Remington G et al (2013) Effects of risperidone and olanzapine dose reduction on cognitive function in stable patients with schizophrenia: an open-label, randomized, controlled, pilot study. Schizophr Bull 39(5):993-998. https://doi. org/10.1093/schbul/sbt090

14. Zhou Y, Li G, Li D et al (2018) Dose reduction of risperidone and olanzapine can improve cognitive function and negative symptoms in stable schizophrenic patients: a single-blinded, 52-week, randomized controlled study. J Psychopharmacol 32(5):524-532. https://doi.org/10.1177/0269881118756062

15. Ran M-S, Weng X, Chan CL-W et al (2015) Different outcomes of never-treated and treated patients with schizophrenia: 14-year follow-up study in rural China. Br J Psychiatry 207(6):495-500. https://doi.org/10.1192/bjp.bp.114.157685

16. Hall W (2012) Harm reduction guide to coming off psychiatric drugs. The Icarus Project and Freedom Center. www.willhall.net/ files/ComingOffPsychDrugsHarmReductGuide2Edonline.pdf

17. Lehmann P (2004) Coming off psychiatric drugs. Successful withdrawal from neuroleptics, antidepressants, lithium, carbamazepine and tranquilizers. Springer, Berlin

18. Wunderink L, Nienhuis FJ, Sytema S et al (2007) Guided discontinuation versus maintenance treatment in remitted first-episode psychosis: relapse rates and functional outcome. J Clin Psychiatry 68(5):654-661

19. Moncrieff J (2006) Does antipsychotic withdrawal provoke psychosis? Review of the literature on rapid onset psychosis (supersensitivity psychosis) and withdrawal-related relapse. Acta Psychiatr Scand 114:3-13

20. Andreasen N, Carpenter W, Kane J et al (2005) Remission in schizophrenia. Proposed criteria and rationale for consensus. Am J Psychiatry 62:441-449

21. Guy W (1976) Clinical global impression. In: ECDEU assessment manual for psychopharmacology, revised (DHEW Publ. No. ADM 76-338). National Institute of Mental Health, Rockville, pp 218-222

22. Kay SR, Fiszbein A, Opler LA (1987) The positive and negative syndrome scale (PANSS) for schizophrenia. Schizophr Bull 13(2):261-275

23. Naber D (1995) A self-rating to measure subjective effects of neuroleptic drugs, relationships to objective psychopathology, quality of life, compliance and other clinical variables. Int Clin Psychopharmacol 10(suppl. 3):133-138
24. Morosini PL, Magliano L, Brambilla L et al (2000) Development, reliability and acceptability of a new version of the DSMIV Social and Occupational Functioning Assessment Scale (SOFAS) to assess routine social functioning. Acta Psychiatr Scand 101(4):323-329

25. Thompson K, Kulkarni J, Sergejew AA (2000) Reliability and validity of a new Medication Adherence Rating Scale (MARS) for the psychoses. Schizophr Res 42(3):241-247

26. Guy U (1976) Abnormal involuntary movement scale. ECDEU assessment manual for psychopharmacology. US Department of Health, Education and Welfare, Washington DC

27. Lingjaerde O, Ahlfors UG, Bech $P$ et al (1987) The UKU side effect rating scale. A new comprehensive rating scale for psychotropic drugs and a cross-sectional study of side effects in neuroleptic-treated patients. Acta Psychiatr Scand Suppl 334:1-100

28. Gardner DM, Murphy AL, O'Donnell H et al (2010) International consensus study of antipsychotic dosing. Am J Psychiatry 167(6):686-693. https://doi.org/10.1176/appi.ajp.2009.09060802

29. Blackwelder WC (1982) "Proving the null hypothesis" in clinical trials. Control Clin Trials 3(4):345-353. https://doi. org/10.1016/0197-2456(82)90024-1

30. Miller R, Pavlo AJ (2018) Two experts, one goal: collaborative deprescribing in psychiatry. Curr Psychiatry Rev 14(1):12-18. https://doi.org/10.2174/1573400514666180531095618

31. Harrow M, Grossman LS, Herbener ES et al (2000) Ten-year outcome: patients with schizoaffective disorders, schizophrenia, affective disorders and mood-incongruent psychotic symptoms. $\mathrm{Br}$ J Psychiatry 177:421-426. https://doi.org/10.1192/bjp.177.5.421

32. Wunderink L, Nieboer RM, Wiersma D et al (2013) Recovery in remitted first-episode psychosis at 7 years of follow-up of an early dose reduction/discontinuation or maintenance treatment strategy: long-term follow-up of a 2-year randomized clinical trial. JAMA Psychiatry 70(9):913-920. https://doi.org/10.1001/jamapsychi atry.2013.19

33. Hui CLM, Honer WG, Lee EHM et al (2018) Long-term effects of discontinuation from antipsychotic maintenance following firstepisode schizophrenia and related disorders: a 10 year follow-up of a randomised, double-blind trial. Lancet Psychiatry 5(5):432442. https://doi.org/10.1016/S2215-0366(18)30090-7 